\title{
First true initial ictal SPECT in partial epilepsy verified by electroencephalography
}

\author{
Jesús Pastor \\ Luis Domínguez-Gadea ${ }^{2}$ \\ Rafael G Sola ${ }^{3}$ \\ Virgilio Hernando 3 \\ María Luisa Meilán ${ }^{4}$ \\ Eva De Dios ${ }^{4}$ \\ José Luis Martínez-Chacón ${ }^{4}$ \\ Marcos Martínez \\ 'Clinical Neurophysiology; \\ ${ }^{2}$ Nuclear Medicine; ${ }^{3}$ Neurosurgery; \\ ${ }^{4}$ Anaesthesiology and Reanimation; \\ Hospital Universitario "La Princesa", \\ Madrid, Spain; ${ }^{5}$ Anaesthesiology and \\ Reanimation, Cardiovascular Unit \\ of Adults, Hospital Ramón y Cajal, \\ Madrid, Spain
}

\begin{abstract}
Drug-resistant epilepsy can sometimes be treated by surgery. In these cases, an accurate identification of the epileptogenic area must be addressed before resection. Ictal SPECT is one of the presurgical evaluations that can be performed, but usually, the increase in the regional cerebral perfusion observed is produced by diffusion of ictal activity. Here we describe a patient studied with v-EEG and foramen ovale electrodes that suffered a seizure after intravenous infusion of etomidate. The sequence of etomidate administration, followed by radiotracer and seizure was good enough for us to suspect that a true initial ictal SPECT was observed. We have implemented a kinetic model with four compartments, previously described (Andersen 1989), in order to estimate the fraction of hydrophilic radiotracer in the brain during the pre-ictal and ictal periods. This model has shown that the fraction of hydrophilic radiotracer during the seizure into the brain would be between $18.9 \%$ and $42.3 \%$ of total infused. We show the first true initial ictal SPECT demonstrated by bioelectrical recordings of the brain activity, obtained by a correct succession of events and compatible with theoretical data obtained from the kinetic model.
\end{abstract}

Keywords: etomidate, foramen ovale electrodes, mathematical model, temporal lobe epilepsy

\section{Introduction}

Epilepsy is one of the most common neurological disorders, affecting almost $0.5 \%-1.0 \%$ of the general population (Theodore et al 2006). Patients usually respond well to pharmacological treatment. However, approximately, $25 \%-30 \%$ of patients are drug-resistant (Engel 1996a). For this group, surgery can be a suitable therapeutic option (Engel 1992, 1996b; Wiebe et al 2001). Surgical treatment requires an exhaustive presurgical evaluation in order to identify the epileptogenic area and minimize the side effects of the operation. Presurgical evaluation usually includes, among others (Pastor et al 2005; Sola et al 2005), electroencephalography (EEG), videoelectroencephalography ( $\mathrm{v}-\mathrm{EEG})$, magnetic resonance imaging (MRI) and single photon emission computerized tomography (SPECT).

Interictal or basal SPECT is a presurgical study not performed systematically in the majority of epilepsy-surgery units because it is affected by a number of drawbacks that limits its results (Henry and Heertum 2003). In order to overcome these problems, some epilepsy-surgery units perform ictal SPECT, in which a radiotracer is intravenously administered after a seizure onset is identified (Spencer et al 2000; Van Paessen et al 2000; Henry and Heertum 2003; Cascino et al 2004). Ictal SPECT has been shown to be an accurate means of localization of temporal lobe seizures when the tracer is injected immediately after the seizure (Catafau 2001; Van Paessen 2004). The localizing information was better when the injection was given soon after seizure onset (Newton et al 1992; Avery et al 1999; Van Paessen et al 2000).

Sometimes, during v-EEG recording and in order to increase the probability of seizures, pharmacologically induced stimulation has been performed using different 
drugs; ie, clonidine, metohexital, etc (Kofke et al 1993; Brockhaus et al 1997; Schmitt et al 1999). Among these, the use of etomidate, a nonbarbiturate hypnotic, short-lived imidazol derivate, has been rare (Ebrahim et al 1986; Modica et al 1990).

In the Hospital La Princesa, currently, we are characterizing the response to etomidate in patients with mesial temporal lobe epilepsy (MTLE) and undergoing evaluation for surgery while they are monitored with v-EEG using foramen ovale electrodes (FOE). It is not our objective to describe this method in detail (see Dominguez-Gadea et al 2006), but to show a fortunate result obtained in the context of that study, describing the case of a patient that suffered a seizure during etomidate infusion after the radiotracer injection for activated SPECT. This allowed us to obtain that which is, to our knowledge, the first true initial ictal SPECT (recorded during the true beginning of the seizure and not during the propagated ictal activity) described in the literature. We have implemented a numerical model previously published (Andersen 1989) for the analysis and discussion.

\section{Case report}

A 14-year-old, right handed girl with a diagnosis of refractory temporal lobe epilepsy (TLE) was referred to the Epilepsy Unit, Hospital Universitario "La Princesa", Madrid, Spain for evaluation and surgical treatment of epilepsy.

Pregnancy and birth were normal. No familial epilepsy history was reported. Seizures began at age 2 years, after a viral vaccination. Nowadays, she complains of daily seizures.

Previously tried anti-epileptic drugs (AED) included phenitoin, carbamacepine, etosuximide, zonisapride, and levetiracetam. At the present time pharmacological treatment included (mg/d): topiramate 300; oxcarbamazepine 1500; valproate 600; and clobazam 10 .

\section{Methods}

Presurgical evaluation, following the previously described La Princesa Hospital protocol (Pastor et al 2005; Sola et al 2005), included neurological and neuropsychological examinations, scalp EEG (Nihon Kohden ${ }^{\circledR}$ ) with 19 electrodes according the 10-20 international system, 1.5 T magnetic resonance imaging (MRI; General Electric ${ }^{\circledR}$ ) and interictal single photon emission computerized tomography (SPECT, General Electric $^{\circledR}$ ) with ${ }^{99 m}$ Tc-D,L-Hexamethylene-propyleneamine oxime $\left.{ }^{99 \mathrm{~m}} \mathrm{Tc}-\mathrm{HmPAO}\right)$ and video-electroencephalography (v-EEG; Easy II Cadwell ${ }^{\circledR}$ ) using 19 scalp electrodes and foramen ovale electrodes (Pastor et al 2006) removing AED from the second to day of recording.
This research was approved by the Ethical Committee of the Hospital la Princesa and informed consent was obtained from the parents because the patient was under 18 years.

SPECT imaging studies were performed using $740 \mathrm{MBq}$ of ${ }^{99 \mathrm{~m}} \mathrm{Tc}-\mathrm{HmPAO}$, with a low-energy high-resolution collimator, simple-head camera (Starcam 3200, General Electric ${ }^{\circledR}$ ) and 96 projections of $22 \mathrm{~s}$ each using a $64 \times 64$ matrix. Slices were reconstructed by filtered back projection using a Butterworth filter (order 10 with a 0.6 cut-off).

$\mathrm{V}$-EEG recording was performed with a bandwidth of $1-70 \mathrm{~Hz}$ for scalp electrodes and $1-100 \mathrm{~Hz}$ for FOE recording, with a sampling rate of $200 \mathrm{~Hz}$.

Etomidate (Janssen-Cilag ${ }^{\circledR}$ ) was intravenously applied in the resting condition, with the patient resting in bed, face up, under continuous supervision of an expert anesthesiologist at a dose of $0.1 \mathrm{mg} / \mathrm{kg}$. Perfusion was performed in approximately $1 \mathrm{~min}$. Electrocardiogram and capillary oxygen saturation (pulsioximetry) were continuously monitored throughout the process. Immediately after the etomidate infusion, a bolus of ${ }^{99 \mathrm{~m}} \mathrm{Tc}-\mathrm{HmPAO}$ was intravenously injected. Brain SPECT was acquired in the $30 \mathrm{~min}$ after the complete recovery of the patient.

\section{Kinetic model}

To analyze the theoretical kinetic behavior of ${ }^{99 m} \mathrm{Tc}-\mathrm{HmPAO}$, we used a numerical model including four compartments (see Figure 2B) and four parameters (Andersen 1989). It is important to keep in mind that the kinetic parameters are taken from biological data, but not adjusted for our patient. The basic assumption was that ${ }^{99 \mathrm{~m}}$ Tc-HMPAO behaves according to the microsphere model and exhibits high rate extraction $(76 \%)$ by the brain tissue during the first microcirculatory pass (Lassen et al 1988). Numerical solutions of the model were obtained by developing a home-made program using the Runge-Kutta method for solving differential equations, implemented in MATLAB R2007a (MathWorks, Natic, USA) and running on a personal computer (Pentium IV, 1 Gb RAM).

\section{Results}

The scalp EEG showed the presence of frequent right temporal sharp waves, usually grouped in patterns, intermingled with irregular slow waves. MRI was normal and interictal SPECT showed inconclusive results, with left temporal antero-mesial hypoperfusion doubtful.

Etomidate was intravenously applied by an anesthesiologist (MLM) whereas the patient stayed in the v-EEG monitoring unit. Ten seconds later, ${ }^{99 \mathrm{~m}} \mathrm{Tc}-\mathrm{HmPAO}$ was iv perfused (LD-G) in order to obtain a pharmacologically activated SPECT. Fifty 
two seconds after ${ }^{99 \mathrm{~m}} \mathrm{Tc}-\mathrm{HmPAO}$ injection, a seizure begun with head version to left, followed by left arm hypertony, oral automatisms and mild disconnection. Fifty-two seconds thereafter, the seizure finished and the patient recovered consciousness. No stupor or paralysis was observed, but a mild disorientation and agitation persisted for 25 seconds.

$\mathrm{v}$-EEG recording showed a beta/gamma low voltage pattern in the right posterior mesial area (right FO electrodes \#1 to \#3), as we can observe in Figure 1. A focal desynchronization was observed in the right temporo-occipital area of the scalp.

Injection of the radiotracer began immediately after etomidate and $52 \mathrm{sec}$. before a seizure started. A localized region of intense hyperperfusion was observed only in the right posterior mesial temporal lobe (Figure 2A, arrow). This region fits well with right FOE 1-3, which were involved in the origin of the seizure. No changes were observed in other regions compared with interictal SPECT performed after a 24-h seizure-free period.

The numeric simulation of the model (see Figure 2C) showed that, in theory, at least, the hydrophilic brain concentration at the beginning of the seizure was approximately $18.9 \%$ of the total radiotracer injected, with only $36.6 \%$ remaining lipophilic in the blood. At the conclusion of the seizure, the theoretical brain hydrophilic concentration was
$42.3 \%$ and only $15.3 \%$ of the radio-tracer persisted in its lipophilic form in the blood. This implies that practically all the available ${ }^{99 \mathrm{~m}} \mathrm{Tc}-\mathrm{HmPAO}$ had been extracted by the end of seizure. Of course, we are conscious that these results only show the compatibility between the theoretical model and the time course of events observed in our patient.

\section{Discussion}

This paper is the first report of an instance where the authors have knowledge of a true-early ictal SPECT, with the radiotracer injection performed at an adequate instant, demonstrated by means of bioelectrical recording.

An intense increase in regional cerebral blood flow is one of the hallmarks of partial-onset seizures. This is probably due to an increase in regional synaptic activity and changes in neurotransmission. Usually, the ${ }^{99 \mathrm{~m}} \mathrm{Tc}-\mathrm{HMPAO}$ infusion is performed briefly after the seizure starts and, moreover, a delay must be overcome before the radiotracer gets to the brain. So, ictal SPECT, although it is the most sensitive and reliable neuroimaging procedure for delineating the epileptogenic area in patients with medically intractable epilepsy (Bernal and Altman 2003), probably shows an increase in regional cerebral perfusion that is surely related to the seizure (Van Paesschen 2004), but is almost certainly indicating propagation from the area of ictal onset.

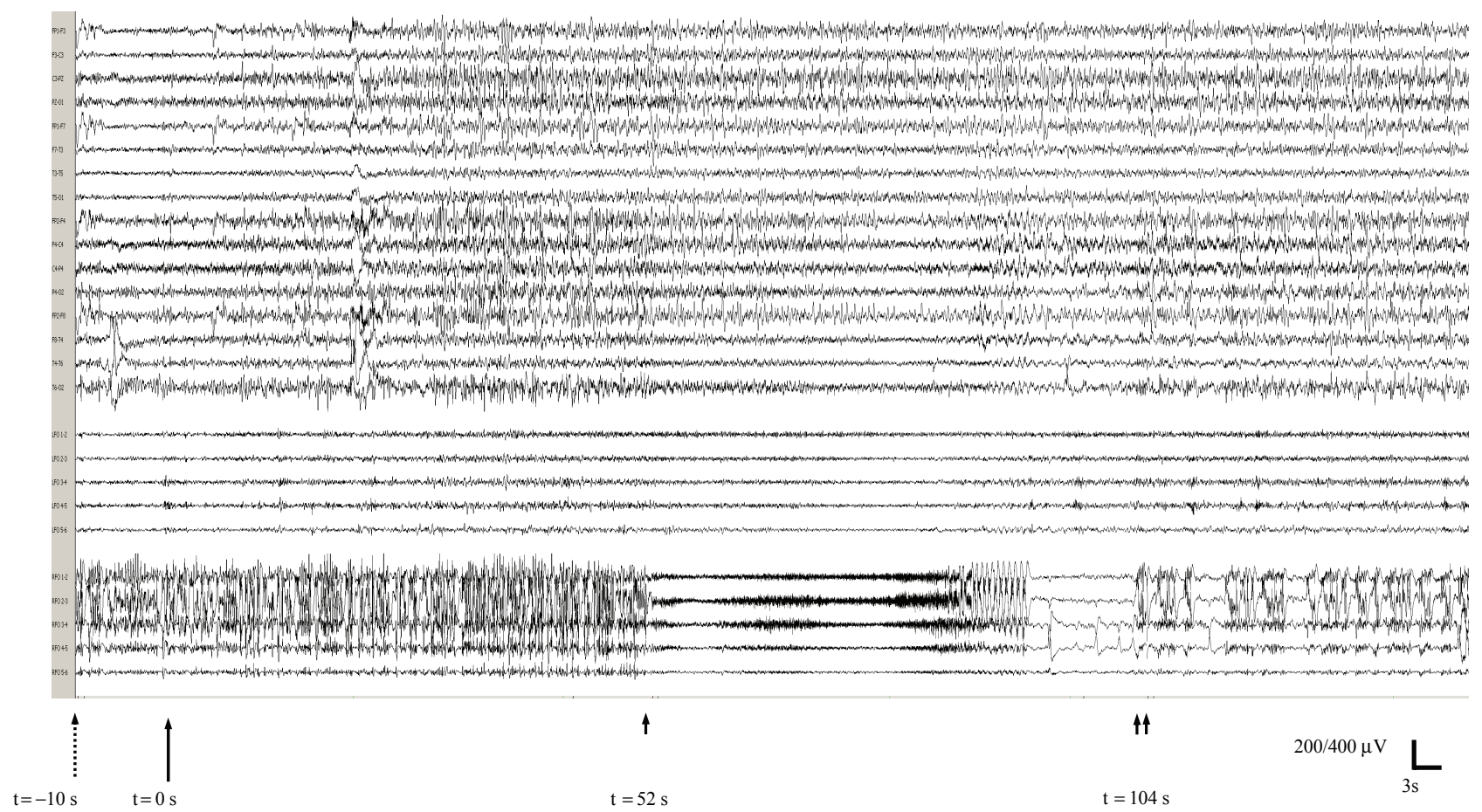

Figure I Recording of v-EEG during administration of etomidate and the subsequent seizure observed. Discontinuous arrow marks the end of etomidate infusion. Continuous arrow shows the ${ }^{99 \mathrm{~m}} \mathrm{Tc}-\mathrm{Hm} \mathrm{mAO}$ infusion. Arrowhead indicates the start of seizure and double arrowhead marks the end of it. 
A
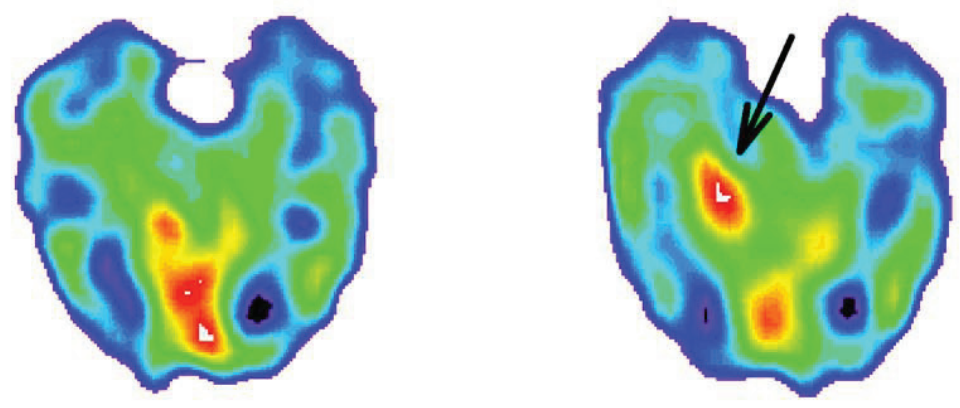

B

Blood

Brain

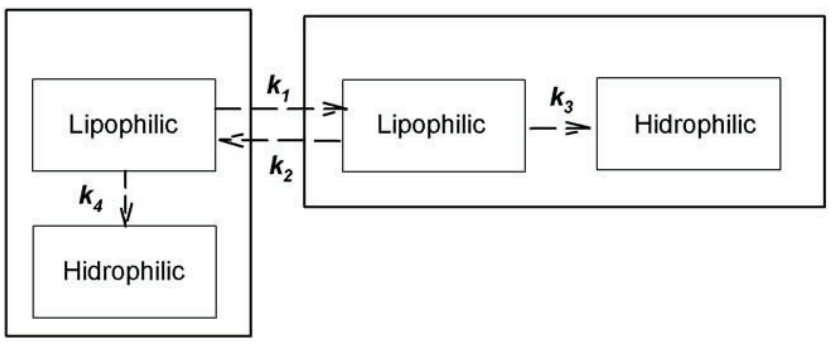

C

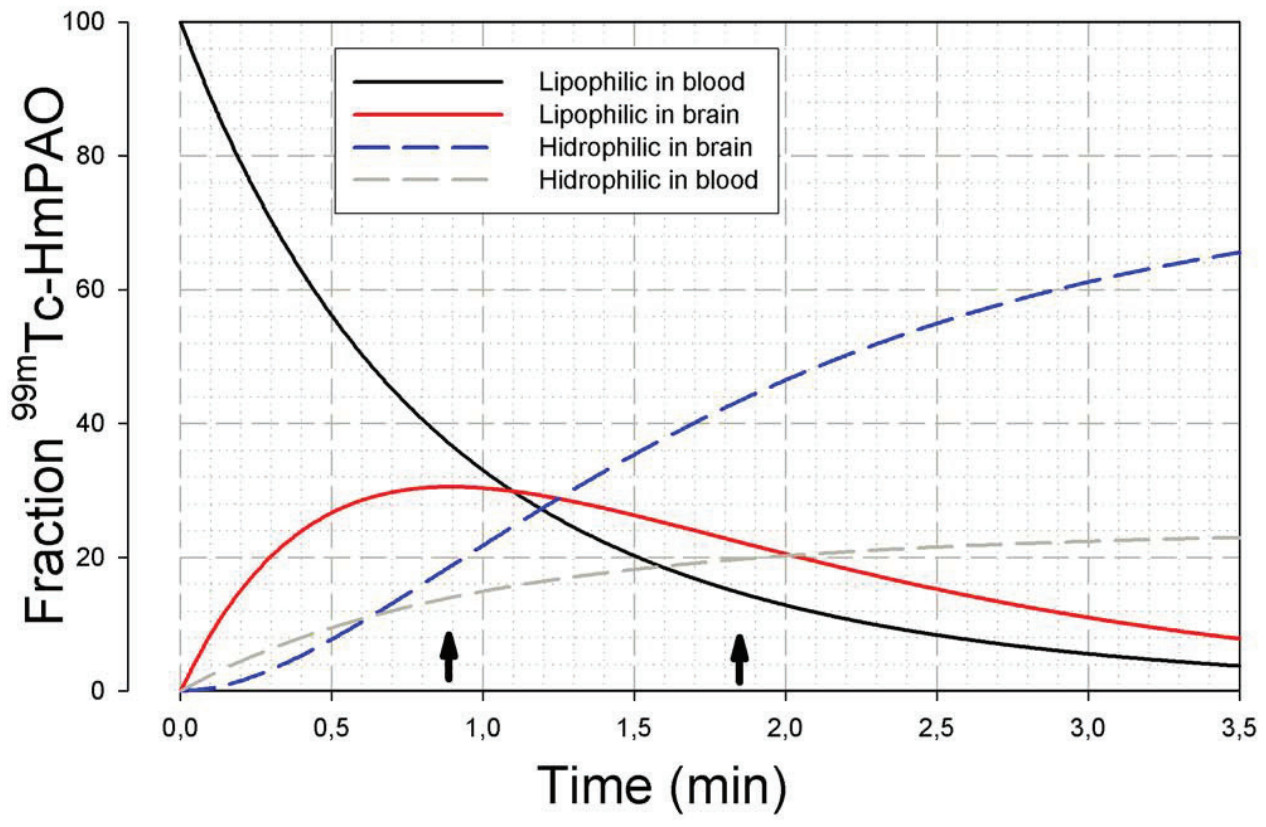

Figure 2 SPECT results and mathematical model of blood-brain ${ }^{99 m} \mathrm{Tc}-\mathrm{HmPAO}$ distribution. A) Basal (left) and etomidate-activated (right) SPECT recordings. The arrow shows the hyperperfusion on the right temporo-occipital area (around fusiform and lingual gyri) that corresponds to the most occipital FO electrodes. B) Kinetic model for numerical solution of distribution for lipophilic and hydrophilic ${ }^{99 \mathrm{~m}} \mathrm{Tc}-\mathrm{HmPAO}$ in blood and brain, according to a four-compartment model. The first-order rate constants are $\left(\mathrm{min}^{-1}\right) k_{1}=0.81 ; k_{2}=0.35 ; k_{3}=0.92$ and $k_{4}=0.25$-Andersen 1989; C) Kinetic evolution of the model during the first 3.5 min after ${ }^{99 m} \mathrm{Tc}-\mathrm{HmPAO}$ iv perfusion that shows the distribution in the different compartments; the broken-blue line represents the evolution of intracellular hydrophilic radio-tracer.The first arrow (from left) marks the start of seizure and the second one indicates the end. 
What is more important, if the delay between seizure onset and injection is big enough, a false lateralization or localization on ictal SPECT due to the "postictal switch" phenomenon can be observed (Newton et al 1992). Thus, the early injection of radiotracer has been considered the most important factor for seizure localization.

Taking into account that a majority of the radiotracer will be taken up in the first pass and that it takes around 30 seconds for the tracer to reach the brain, we can reasonably assume that a small amount of the ligand $(<19 \%)$ probably would be absorbed previous to the seizure onset (pre-ictal state). However, as we can observe from Figure 2C, the most important amount of radiotracer will be taken up by the brain during the seizure (period between both arrows).

The safest and most reproducible way to control the moment of injection could be through the use of a pharmacological activation of the seizures. However, this possibility is far from being ready to use (Diekman et al 1998; Kirchberger et al 1998). In the model that we are developing in order to overcome that problem, the infusion of etomidate induces important changes in the cerebral regional perfusion, but usually with no associated seizures (Domínguez-Gadea et al 2007). Indeed, this case was the only one presenting a crisis out of 46 patients.

In summary, we are conscious that the case reported here was a lucky occurrence, in which the correct order of events allowed to us to demonstrate that an increase in regional cerebral perfusion would be produced in the cerebral area that started the seizure. The systematic methodology in pharmacological activated SPECT with etomidate could produce reproducible and consistent results even without pharmacologically activated seizures. However, more studies are needed before this point will be definitely established.

\section{Acknowledgments}

We thank American Journal Experts LLC for help with the English edition. This work was supported by a grant from the Plan Nacional de Investigación Científica, Desarrollo e Innovación Tecnológica (I+D+I), Instituto de Salud Carlos III, Subdirección General de Evaluación y Fomento de la Investigación PI060349.

\section{References}

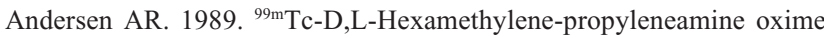
(99mTc-HMPAO): basic kinetic studies of a tracer of cerebral blood flow. Cerebrovasc Brain Metab Rev, 1:288-318.

Avery RA, Spencer SS, Spanaki MV, et al. 1999. Effect of injection time on postictal SPECT perfusion changes in medically refractory epilepsy. Eur J Nucl Med, 26:830-6.

Bernal B, Altman NR. 2003. Evidence-based medicine: neuroimaging of seizures. Neuroimaging Clin N Am, 13:211-24.
Brockhaus A, Lehnertz K, Wiebruch C, et al. 1997. Possibilities and limitations of magnetic source imaging of methohexital-induced epileptiform patterns in temporal lobe epilepsy patients. Electroencephalog Clin Neurophysiol, 102:423-36.

Cascino GD, So EL, Buchhalter JR, et al. 2004. The current place of single photon emission computed tomography in epilepsy evaluations. $\mathrm{Neu}$ roimaging Clin N Am, 14:553-61.

Catafau AM. 2001. Brain SPECT in clinical practice. Part I: Perfusion. J Nucl Med, 42:259-71.

Diekman V, Becker W, Jürgens R, et al. 1998. Localisation of epileptic foci with electric, magnetic and combined electromagnetic models. Electroencephalogr Clin Neurophysiol, 106:297-313.

Domínguez-Gadea L, Pastor J, Meilán ML, et al. 2007. SPECT de perfusión cerebral con etomidato en el estudio prequirúrgico de la epilepsia temporal. Rev Esp Med Nucl, 26:28.

Ebrahim ZY, DeBoer GE, Luders H, et al. 1986. Effect of etomidate on the electroencephalogram of patients with epilepsy. Anesth Anal, 65:1004-6.

Engel J Jr. 1992. Recent advances in surgical treatment of temporal lobe epilepsy. Acta Neurol Scand, 140(Suppl 5):71-80.

Engel J Jr. 1996a. Introduction to temporal lobe epilepsy. Epilepsy Res, 26:141-50.

Engel J Jr. 1996b. Current concepts: surgery for seizures. $N$ Engl J Med, 10:647-52.

Henry TR, van Heertum RL. 2003. Positron emission tomography and single photon emission computed tomography in epilepsy care. Semin Nucl Med, 33:88-104.

Kirchberger K, Schmitt H, Hummel C, et al. 1998. Clonidine- and Methohexital-induced epileptiform discharges detected by magnetoencephalography (MEG) in patients with localization-related epilepsies. Epilepsia, 39:1104-12.

Kofke WA, Dasheiff RM, Dong ML, et al. 1993. Anaesthetic care during thipental test to evaluate epileptic patients for surgical therapy. $J \mathrm{Neu}$ rosurg Anaesthesiol, 5:164-70.

Lassen NA, Andersen AR, Friberg L, et al. 1988. The retention of ( $\left.{ }^{99 \mathrm{~m}} \mathrm{Tc}\right)$ d,1-HM-PAO in the human brain after intracarotid bolus injection: a kinetic analysis. J Cereb Blood Flow Metab, 8:13-20.

Modica PA, Templehoff R, White PF. 1990. Pro-and anticonvulsant effects of anesthetics. Anesth Analg, 70:433-44.

Newton MR, Berkovic SF, Austin MC, et al. 1992. Postictal switch in blood flow distribution and temporal lobe seizures. J Neurol Neurosurg Psychiatr, 55:891-4.

Pastor J, de la Prida LM, Hernando V, et al. 2006. Voltage sources in mesial temporal lobe epilepsy recorded with foramen ovale electrodes. Clin Neurophysiol, 117:2604-14.

Pastor J, Hernando-Requejo V, Dominguez-Gadea L, et al. 2005. Impact of experience on improving the surgical outcome in temporal lobe epilepsy. Rev Neurol, 4:709-16.

Schmitt H, Druschky K, Hummel C, et al. 1999. Detection of an epileptic mirror focus after oral application of clonidine. Br J Anaesth, $83: 349-51$.

Sola RG, Hernando-Requejo V, Pastor J, et al. 2005. Pharmacoresistant temporal-lobe epilepsy. Exploration with foramen ovale electrodes and surgical outcomes. Rev Neurol, 41:4-16.

Spencer SS, Bautista RE. 2000. Functional neuroimaging in localization of the ictal onset zone. Adv Neurol, 83:285-96.

Theodore WH, Spencer SS, Wiebe S, et al. 2006. Epilepsy in North America: a report prepared under the auspices of the global campaign against epilepsy, the International Bureau for Epilepsy, the International League Against Epilepsy, and the World Health Organization. Epilepsia, 47:1700-22.

Van Paesschen W, Dupont P, van Heerden B, et al. 2000. Self-injection ictal SPECT during partial seizures. Neurology, 54: 1994-1997.

Van Paesschen W. 2004. Ictal SPECT. Epilepsia, 45(Suppl 4):35-40.

Wiebe S, Blume WT, Girvin JP, et al. 2001. Effectiveness and Efficiency of Surgery for Temporal Lobe Epilepsy Study Group. A randomized, controlled trial of surgery for temporal-lobe epilepsy. $N$ Engl J Med, 5: $311-8$. 
